\title{
Investigating the transverse motion of a pneumatic shock exciter using two different anvil mounting configurations
}

\author{
Christiaan S. Veldman ${ }^{1}$ \\ ${ }^{1}$ National Metrology Institute of South Africa, CSIR Campus, Brummeria, Pretoria, 0184, South Africa
}

\section{ABSTRACT}

This paper describes novel design changes to the accelerometer mounting support of a commercial pneumatic shock exciter, with the aim of reducing the transverse motion the accelerometer is subjected to during shock excitation. The author describes the mounting support supplied by the manufacturer, the design changes made and the measurement data to compare the transfer motions recorded using two different mounting designs.

\section{Section: RESEARCH PAPER}

Keywords: transverse motion; shock calibration; shock excitation; accelerometer; pneumatic

Citation: Christiaan Smith Veldman, Investigating the transverse motion of a pneumatic shock exciter using two different anvil mounting configurations, Acta IMEKO, vol. 10, no. 2, article 28, June 2021, identifier: IMEKO-ACTA-10 (2021)-02-28

Section Editor: Gustavo Ripper, INMETRO, Brazil

Received October 1, 2020; In final form February 15, 2021; Published June 2021

Copyright: This is an open-access article distributed under the terms of the Creative Commons Attribution 3.0 License, which permits unrestricted use, distribution, and reproduction in any medium, provided the original author and source are credited.

Corresponding author: Ian C.S. Veldman, e-mail: CSVeldman@NMISA.org

\section{INTRODUCTION}

Shock measurements [1], [2] have been performed internationally for a long time. In meeting the requirement to provide traceability for the International System of Units (SI), National Metrology Institutes (NMIs) and commercial companies have developed various calibration procedures utilising different shock exciters (SE) [3]-[9]. The operating principal of the SE depends largely on the intended peakacceleration level. For low-level shocks, acceleration levels of $\approx 10 \mathrm{~m} / \mathrm{s}^{2}$, the 'drop ball' or pendulum method is used. For medium-level shocks, acceleration levels in the range of $50 \mathrm{~m} / \mathrm{s}^{2}$ to $10 \mathrm{~km} / \mathrm{s}^{2}$, a hammer-and-anvil method is employed (rigid body movement of an anvil), using a loaded spring or pneumatic system as the force applicator [10], [11]. For high-shock levels, acceleration levels up to $100 \mathrm{~km} / \mathrm{s}^{2}$, a Hopkinson bar (shock propagation inside a long, thin bar) is used as the shock exciter [8], [10], [11].

As part of its programme of work, the International Organization for Standardization's Technical Committee (ISO/TC) 108 developed parts 13 and 22 in the ISO 16063 series of standards [10], [11]. Part 13 of the standard specifies primary calibration procedures to determine the shock sensitivity of accelerometers, while part 22 of the standard specifies secondary calibration procedures to determine the shock sensitivity of accelerometers by comparison with a reference transducer.
These procedures have been implemented by various NMIs [3], [5], [6], [9]. The procedures relating to part 13, the implementation thereof and the NMIs' calibration measurement capabilities were validated during the first official international shock comparison, CCAUV.V-K4 [12].

A prominent component in the estimation of the standard uncertainty (UoM) in accelerometer calibrations is the effect of transverse motion (TM) [13]-[16]. As we are dealing with mechanical systems, this component of the UoM cannot be reduced to zero. In practice, the TM uncertainty contribution is a result of two factors:

1) the physical TMs

2) the transverse sensitivity of the accelerometer being calibrated [17]-[21].

As a result, care is taken to reduce the TM of the exciter as much as possible. In the case of the rigid body movement of an anvil system, this is generally achieved by using air-bearing guides.

In this research paper, the author investigates two anvil support configurations implemented in a commercial pneumatic $\mathrm{SE}$ system. In Section 2, the system in question is described, outlining the two mounting configurations and the reduced TM envisaged by the implementation of the proposed changes. Section 3 deals with the methods and instrumentation used to perform the TM measurements, which are then reported and discussed in Section 4. Section 3 also describes two different 
methodologies for considering the results. The author then draws some conclusions in Section 5.

\section{ANVIL MOUNTING DESIGN}

The accelerometer shock sensitivity calibration system implemented at South Africa's NMI, NMISA, utilises a SPEKTRA SE-201 pneumatic exciter. This exciter uses two anvil systems to cover the complete manufacturer's specified acceleration range from $50 \mathrm{~m} / \mathrm{s}^{2}$ to $100 \mathrm{~km} / \mathrm{s}^{2}$. An air-bearing anvil unit is used for the acceleration sub-range from $50 \mathrm{~m} / \mathrm{s}^{2}$ to $2,500 \mathrm{~m} / \mathrm{s}^{2}$. For the high-shock (HS) acceleration range, a lightweight aluminium anvil system is used, covering the acceleration range from $2 \mathrm{~km} / \mathrm{s}^{2}$ to $100 \mathrm{~km} / \mathrm{s}^{2}$.

For HS, the accelerometer is mounted onto the anvil using a mounting stud. This accelerometer/anvil system forms the rigid body (RB) and is suspended (held in place) using a rubber band (O-ring) in a longitudinal configuration (Mount 1). The O-ring position is shown graphically as the light blue line in Figure 1. The O-ring is hooked around two aluminium pillars mounted on either side of the exciter-system base plate. The pillars have a groove to keep the O-ring in place. This arrangement keeps the $\mathrm{RB}$ gently at rest, waiting for the hammer strike. In the present study, we define this axial line as the $\mathrm{Y}$ axis.

As can be seen in Figure 1, this arrangement results in two major and opposite tension forces, $\mathrm{T}_{\mathrm{S}, 1.1}$ and $\mathrm{T}_{\mathrm{S}, 1.2}$. There also exist two opposite tension forces, $T_{\mathrm{S}, 1.3}$ and $T_{\mathrm{S}, 1.4}$, which are much smaller than, and perpendicular to, $\mathrm{T}_{\mathrm{S}, 1.1}$ and $\mathrm{T}_{\mathrm{S}, 1.2}$. When the dominant forces are in line with the $\mathrm{Y}$ axis, this longitudinal configuration will restrain movement along the $\mathrm{Y}$ axis, with substantially more freedom of movement (less constraint) along the $\mathrm{X}$ axis.

Mount 2, a novel, inexpensive design modification for the mounting configuration, was implemented in this study. The red line in Figure 1. illustrates the configuration for Mount 2. The configuration results in four, almost equal tension forces. Two opposing tension forces, $\mathrm{T}_{\mathrm{S}, 2.1}$ and $\mathrm{T}_{\mathrm{S}, 2.2 \text {, are perpendicular to }}$ $\mathrm{T}_{\mathrm{S}, 2.3}$ and $\mathrm{T}_{\mathrm{S}, 2.4}$. The resulting net force action on the $\mathrm{RB}$ results in a more uniform and balanced constraint in both $\mathrm{X}$ and $\mathrm{Y}$ directions.

The process for modifying the SE high-shock mount from Mount 1 to Mount 2 is very simple and very low cost. The

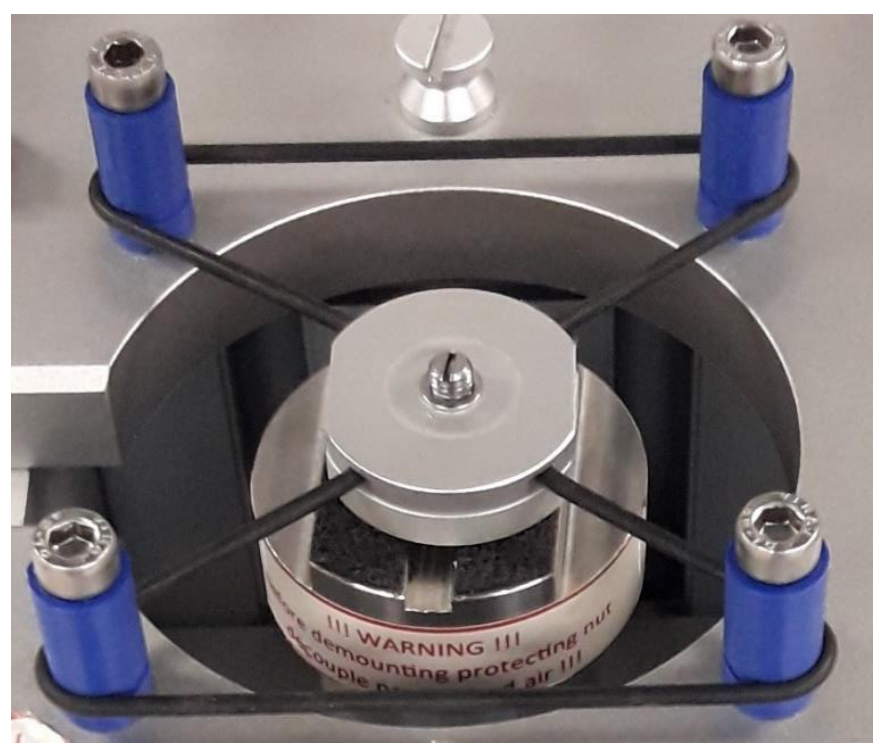

Figure 2. The new design layout without the triaxial accelerometer for clarity.

supplied O-ring is unhooked from the two suspension pillars and replaced with an O-ring with a larger circumference, $\varnothing \approx 100 \mathrm{~mm}$. The HS top safety plate is secured using four Allen cap screws and four 20-mm-long spacers with a diameter of $12 \mathrm{~mm}$. The four Allen cap screws are removed one by one, and the O-ring is hooked over the aluminium spacer. Once the Oring is anchored around all four mounting posts supporting the top plate, the RB is held in position by four almost equal length and perpendicular rubber springs (a section of an O-ring) in an ' $\mathrm{X}$ ' configuration, as shown in Figure 2. These four sections of the O-ring create almost equal and opposite perpendicular forces, which are applied to the RB at rest and in motion, reducing the resulting TM.

To ensure that the O-ring stays in place (does not move upward because of the shocks), the aluminium spacers were replaced with spacers containing a groove, into which the O-ring was slotted. These spacers (Figure 3) were designed using FreeCAD ${ }^{\odot}$ software and manufactured using $3 \mathrm{D}$-printing technology.

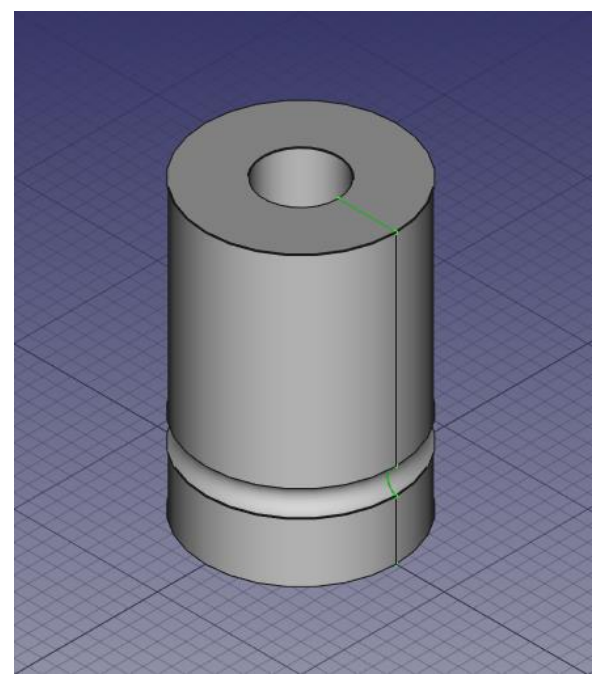

Figure 3. Example of a 3D-printed support pillar to replace the aluminium

pillar.
Figure 1. A pictorial representation, showing force vectors, of the two O-ring design layouts investigated. Blue: original design, S1. Red: new design, S2. 


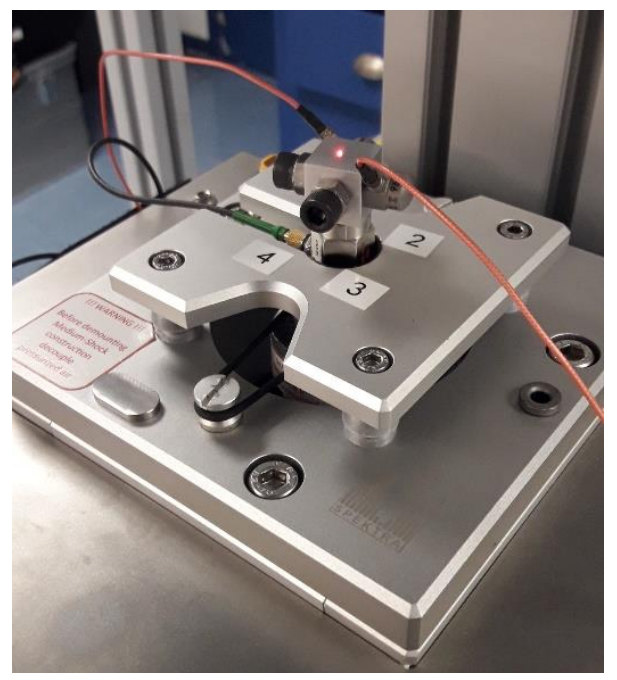

Figure 4. Measurement configuration showing the two accelerometers to measure acceleration in $\mathrm{X}$ and $\mathrm{Y}$ and the laser spot to measure acceleration in $\mathrm{Z}$ in the Mount 1 configuration.

\section{TRANSVERSE MOTION MEASUREMENTS}

The TM (acceleration along the $\mathrm{X}$ and $\mathrm{Y}$ axes) was measured using a bi-axial accelerometer, while the principal acceleration ( $\mathrm{Z}$ axis) was measured using laser interferometry. The measurement setup is shown in Figure 4.

\subsection{Bi-axial accelerometer}

A bi-axial $(\mathrm{X}-\mathrm{Y})$ accelerometer was constructed using a $15 \mathrm{x}$ 15 x 15 mm aluminium block. Two Endevco model 7259-100 IEPE accelerometers were stud mounted perpendicular to each other to measure acceleration in the $\mathrm{X}$ and $\mathrm{Y}$ axes. These accelerometers have a nominal sensitivity of $10 \mathrm{mV} /\left(\mathrm{m} / \mathrm{s}^{2}\right)$ over a wide frequency range, typically $5 \mathrm{~Hz}$ to $30 \mathrm{kHz}$, with a specified peak acceleration of $500 \mathrm{~m} / \mathrm{s}^{2}$. Even though the TM was to be measured for shock levels of up to $40 \mathrm{~km} / \mathrm{s}^{2}$, the expected peak TM did not exceed the specified acceleration range of the accelerometer.

The construction of the bi-axial accelerometer was completed with the attachment of an M5 Allen cap screw with a nut on the opposing side of each accelerometer. These Allen cap screws with nuts were added to allow for a centre of gravity (CG) adjustment to the bi-axial accelerometer. The CG was adjusted to be as close to the centre of the aluminium cube as possible.

\subsection{Measurement methodology}

Measurement data were collected using a National Instruments PXI unit (NI-PXI). The PXI unit was fitted with three dual channel data acquisition units (DAQ). One DAQ sampled the interferometer I and Q signals used for measuring the shock peak acceleration. The second DAQ was used to sample the output signal of the $\mathrm{X}$ - and $\mathrm{Y}$-axis accelerometers, used to measure the transverse acceleration, while the third DAQ was used to trigger (start) each sampling event. The sampling of the three DAQ units was synchronised using the NI niTClk system, which enabled the data sampling to be time synchronised. The calculated average of a set of five measurements was taken as the measurement result for each measurement point (acceleration level).

The resulting transverse acceleration was calculated as

$a_{r}=\sqrt{a_{X}^{2}+a_{Y}^{2}}$

where $a_{r}$ is the resulting transverse acceleration, $a_{X}$ the measured acceleration in the $\mathrm{X}$ direction and $a_{Y}$ the measured acceleration in the $\mathrm{Y}$ direction. An example of a measured transverse acceleration is shown in Figure 5. The TM reported and evaluated was the peak transverse acceleration relative to the peak acceleration of interest, the peak acceleration of interest being the peak acceleration along the $\mathrm{Z}$ axis. The relative TM was calculated as

$a_{T}=\frac{a_{r}}{a_{Z}}$

where $a_{T}$ is the relative transverse acceleration (RTA), $a_{r}$ is the resulting TM, calculated using (1), and $a_{Z}$ is the peak acceleration along the $\mathrm{Z}$ axis. Measurements were performed over the acceleration range of $5 \mathrm{~km} / \mathrm{s}^{2}$ to $40 \mathrm{~km} / \mathrm{s}^{2}$.

The measurement results revealed a time delay between the reference acceleration (measured by the laser interferometer) and the TM $\left(a_{T}\right)$ peak. The time delay between the two peaks can be

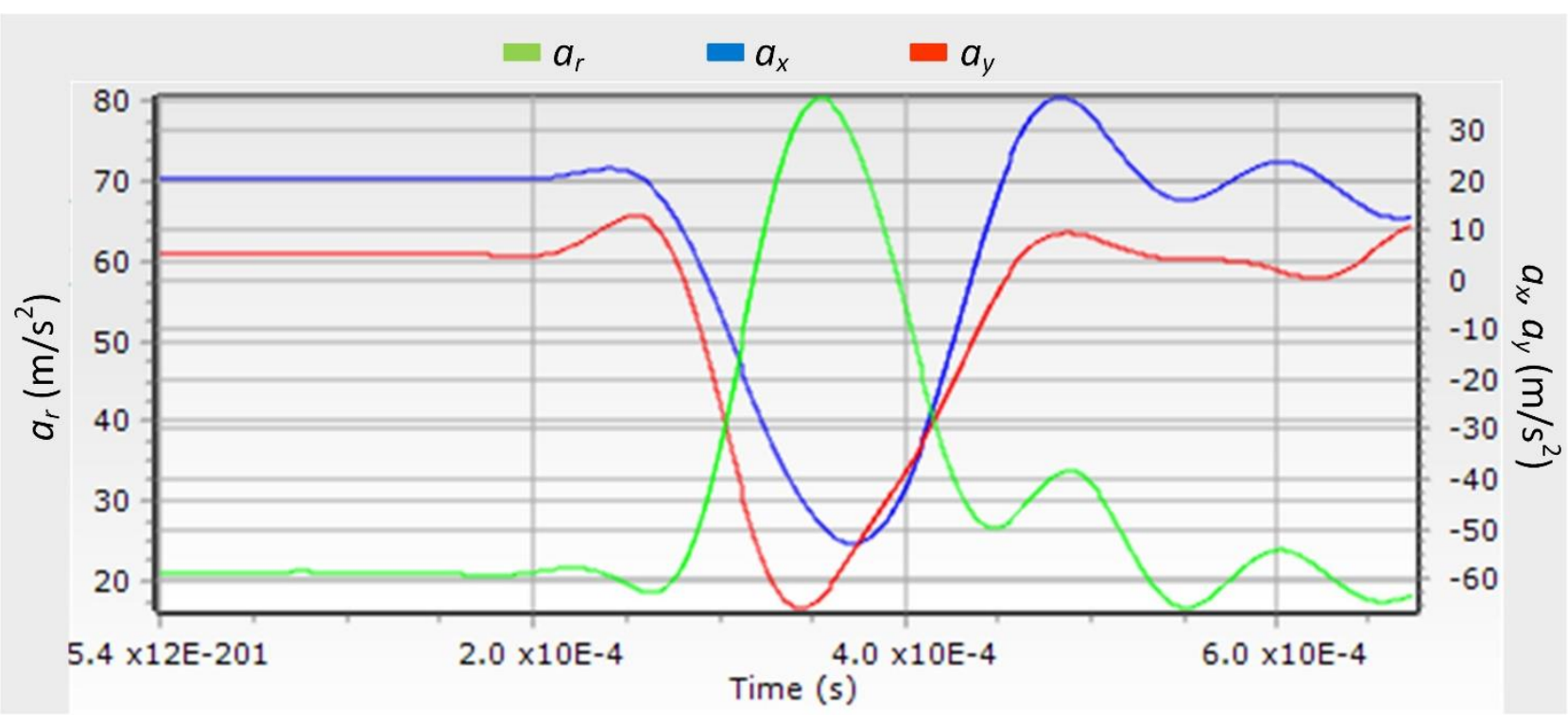

Figure 5. Example of a measured TM during shock measurements, showing $a_{\mathrm{x}}, a_{\mathrm{y}}$ and the resulting $a_{\mathrm{r}}$. 
Table 1. Relative TM measurement results at the time of the Z-axis peak.

\begin{tabular}{ccc}
\hline $\begin{array}{c}\text { Peak } \\
\text { Acceleration }\end{array}$ & $\begin{array}{c}\boldsymbol{a}_{\mathrm{T}} \\
\text { Mount 1 } \\
\text { @ Ref peak }\end{array}$ & $\begin{array}{c}\boldsymbol{a}_{\mathrm{T}} \\
\text { Mount 2 } \\
\text { @ Ref peak }\end{array}$ \\
\hline$\left(\mathbf{k m} / \mathbf{s}^{\mathbf{2})}\right.$ & $\mathbf{( \% )}$ & $\mathbf{( \% )}$ \\
5 & 1.9 & 1.1 \\
10 & 1.4 & 1.2 \\
20 & 0.9 & 0.8 \\
40 & 0.6 & 0.5 \\
\hline
\end{tabular}

seen in Figure 6. In view of this, two different peak RTA evaluation methodologies were considered and reported on:

1) time-delayed peak transverse acceleration $\left(a_{T}\right)$, which is the peak TM measured during the complete sampling time,

2) transverse acceleration at the time of the reference acceleration peak, which is the transverse acceleration level at the time (instance) of the Z-axis acceleration.

\section{MEASUREMENT RESULTS}

The RTA was measured at 5, 10, 20 and $40 \mathrm{~km} / \mathrm{s}^{2}$. Measurements were performed using both mounting support designs described in Section 2, Mount 1 and Mount 2. Furthermore, the RTA was determined at the two instances in time described in Section 3.2. Time instance one at the RTA peak and time instance two synchronised with the Z-axis peak, hence referred to as the@ Ref peak. The results recorded for the two different mounts under investigation, as calculated using@Ref, are reported in Table 1, with the results recorded for the two different mounts calculated at the RTA peak reported in Table 2.

For accelerometer sensitivity calibration, the metrologist is concerned with the TM severity in the presence of an accelerometer's transverse sensitivity. During rectilinear excitation, it can be assumed that TM is present while the exciter is in motion. Although this is true during the shock excitation described in this research paper, the metrologist is only concerned with the RTA the accelerometer is subjected to during the time/period of the shock pulse.

The data reported in Table 1 reveals that the implementation of Mount 2 marginally improves the RTA compared to Mount 1,
Table 2. Relative TM measurement results at the time of the RTA peak.

\begin{tabular}{ccc}
\hline $\begin{array}{c}\text { Peak } \\
\text { Acceleration }\end{array}$ & $\begin{array}{c}\boldsymbol{a}_{\mathrm{T}} \\
\text { Mount 1 } \\
\text { RTA peak }\end{array}$ & $\begin{array}{c}\boldsymbol{a}_{\mathrm{T}} \\
\text { Mount 2 } \\
\text { RTA peak }\end{array}$ \\
\hline$\left(\mathbf{k m} / \mathbf{s}^{\mathbf{2})}\right.$ & $\mathbf{( \% )}$ & $\mathbf{( \% )}$ \\
5 & 3.4 & 1.8 \\
10 & 2.6 & 2.0 \\
20 & 1.8 & 1.2 \\
40 & 1.6 & 1.5 \\
\hline
\end{tabular}

with the RTA improvement being more significant at lower shock acceleration levels. At $5 \mathrm{~km} / \mathrm{s}^{2}$, the RTA is reduced by about $42 \%$, which translates into the same percentage reduction in the RTA uncertainty contribution. As the shock level increases from $5 \mathrm{~km} / \mathrm{s}^{2}$ to $40 \mathrm{~km} / \mathrm{s}^{2}$, the RTA reduces from $1.9 \%$ to $0.6 \%$ using Mount 1, while the RTA reduces from $1.1 \%$ to $0.5 \%$ using Mount 2. The data reported supports the prediction of reduced TM through the novel anvil suspension design change.

In consideration of the data reported in Table 2, similar trends in the RTA are noted between the two different mountings investigated. The RTA tends to reduce as the shock acceleration level increases. It is noted that the RTA using Mount 2 (' $\mathrm{X}$ ' configuration) is consistently lower than when using Mount 1, with the largest reduction measured at $5 \mathrm{~km} / \mathrm{s}^{2}$. However, it is necessary to consider the data carefully within the conditions of the data assessment.

As pointed out earlier, as the shock level increases, the time difference between the Z-axis shock peak and the RTA peak increases; that is, the RTA peak is reached some time after the principal axis acceleration peak. For shocks with peak acceleration levels above $10 \mathrm{~km} / \mathrm{s}^{2}$, the RTA peak occurred after the end of the sampling time. The aim of the investigation was not to determine the actual peak TM, but the TM that influences the uncertainty in shock sensitivity calibration.

\section{CONCLUSIONS}

The performance with respect to the resulting TM of two different mounting configurations used to mount/support the anvil of a pneumatic SE were investigated. Two different instances of peak TM were also considered, first, at the instance

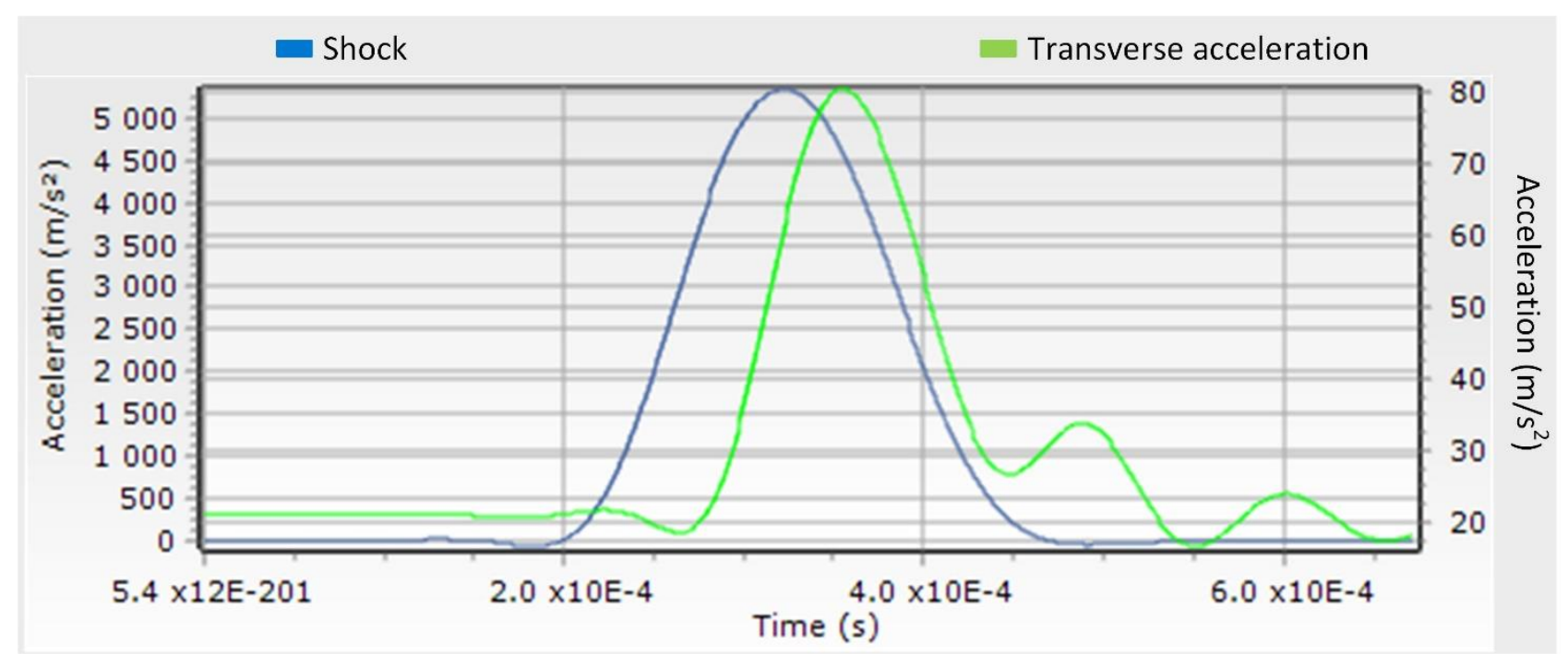

Figure 6. Shock (left vertical axis) and transverse acceleration (right vertical axis) time series. 
in time when the peak acceleration occurs, and second, when the resulting TM $\left(a_{r}\right)$ peak occurs, which is generally some time after the principal axis acceleration peak. In terms of the influence of RTA as an uncertainty contributor, the author focused on the results from the first instance.

The results indicate that the configuration in Mount 2 provides a reduction in TM of almost $50 \%$ at shocks of $5 \mathrm{~km} / \mathrm{s}^{2}$. The performance gain using Mount 2 reduces as the shock acceleration increases to almost 0 for peak shock levels above $40 \mathrm{~km} / \mathrm{s}^{2}$

The benefit (with possible reduced expanded uncertainty of measurement) of implementing the proposed mounting configuration is worth the minimal mechanical changes required to implement it. It should be noted that with this mounting configuration, mounting the accelerometer onto the anvil is a little more time consuming than the manufacturer's original configuration.

\section{REFERENCES}

[1] ISO 16063-1, Methods for the calibration of vibration and shock transducers - Part 11: Basic concepts.

[2] ISO 2041, Mechanical vibration, shock and condition monitoring - Vocabulary.

[3] C. S. Veldman, M. Mutloatse, Establishing primary shock sensitivity capabilities at NMISA, Proc. of Test \& Measurement Conference, 2018.

[4] C. S. Veldman, Implementing a shock calibration system using a vibration exciter and pendulum, NCSLI Measurement March (2014), pp. 40-45.

DOI: $10.1080 / 19315775.2014 .11721672$

[5] H.-J. von Martens, A. Täubner, W. Wabinski, A. Link, H.-J. Schlaak, Traceability of vibration and shock measurements by laser interferometry, Measurement 28 (2000), pp. 3-20. DOI: $10.1016 /$ S0263-2241(00)00003-8

[6] H. Nozato, T. Usuda, A. Oota, T. Ishigami, Calibration of vibration pick-ups with laser interferometry: part IV. Development of a shock acceleration exciter and calibration system, Measurement Science and Technology, Volume 21, Number 6, 2010, pp. 065107 (10 pp.).

DOI: $\underline{10.1088 / 0957-0233 / 21 / 6 / 065107}$

[7] H. Nicklich, M. Brucke, H.-J. von Martens, Design of vibration and shock exciters for calibrations by laser interferometry, Proc. of SPIE 7098, Eighth International Conference on Vibration Measurements by Laser Techniques: Advances and Applications, Ancona, Italy, 17 June 2008, 13 pp.

[8] H. Nicklich, M. Brucke, M. Mende, The need for controlled shocks - a new type of shock exciter allows to apply well defined mechanical shocks, Proc. of the XIXX IMEKO World Congress, Fundamental and Applied Metrology, Lisbon, Portugal, 6-11 September 2009, pp. 1282. Online [Accessed 11 June 2021] https://www.imeko.org/publications/wc-2009/IMEKO-WC2009-TC22-396.pdf

[9] G. B. Micheli, G. P. Ripper, R. S. Dias, C. D. Ferreira, Dispersion analysis of accelerometer shock calibrations by comparison using a new exciter developed at INMETRO, J. Phys. Conf. Ser. 648, $3^{\text {rd }}$
International Congress on Mechanical Metrology, Gramado, Rio Grande do Sul, Brazil, 14-16 October 2015, pp. 648. DOI: $10.1088 / 1742-6596 / 648 / 1 / 012017$

[10] ISO 16063-13, Methods for the calibration of vibration and shock transducers - Part 13: Primary shock calibration using laser interferometry.

[11] ISO 16063-22 Methods for the calibration of vibration and shock transducers - Part 22: Shock calibration by comparison to a reference transducer.

[12] S. Qiao, H. Hongbo, A. Ota, H. Nozato, G. P. Ripper, L. M. M. Mendoza, A. Kozlyakovskiy, C. S. Veldman, T. Bruns, L. Dickinson, Y. B. Lee, Key comparison in the field of acceleration on low intensity shock sensitivity, Metrologia 56 (2019) Tech. Suppl. 09003.

DOI: $10.1088 / 0026-1394 / 56 / 1 \mathrm{a} / 09003$

[13] H. Nozato, A. Oota, T. Ishigami, T. Usuda, The methods for the calibration of vibration pick-ups by laser interferometry: part V. Uncertainty evaluation on the ratio of transducer's peak output value to peak input acceleration in shock calibration, Measurement Science and Technology, 15 November 2011, Volume 22, Number 12, pp. 125109.

DOI: $\underline{\text { 10.1088/0957-0233/22/12/125109 }}$

[14] A. Oota, T. Usuda, H. Nozato, T. Ishigami, T. Kikuchi, Estimation of uncertainty contribution of transverse sensitivity and vibration disturbance on primary accelerometer calibration, Proc. of the XIXX IMEKO World Congress, Fundamental and Applied Metrology, Lisbon, Portugal, 6-11 September 2009, pp. 1282. Online [Accessed 11 June 2021]. https://www.imeko.org/publications/wc-2009/IMEKO-WC2009-TC22-095.pdf

[15] C. S. Veldman, Accelerometer transverse sensitivity calibration; validation and uncertainty estimation, Acta IMEKO 4 (2015) 2, pp. 52-56. DOI: $10.21014 /$ acta imeko.v4i2.209

[16] H. Volkers, T. Beckmann, R. Behrendt, Investigations of reference surface warp at high shock calibrations, IMEKO 22nd TC3, 12th TC5 and 3rd TC22 International Conferences, Cape Town, Republic of South Africa, 3-5 February 2014, 4 pp. Online [Accessed 11 June 2021] https://www.imeko.org/publications/tc22-2014/IMEKOTC22-2014-009.pdf

[17] ISO 16063-31, Methods for the calibration of vibration and shock transducers - Part 31: Testing of transverse vibration sensitivity.

[18] ISO 5347-12:1993 Methods for the calibration of vibration and shock pick-ups - Part12: Testing of transverse shock sensitivity.

[19] T. Usuda, C. Weißenborn, H.-J. von Martens, Theoretical and experimental investigation of transverse sensitivity of accelerometers under multiaxial excitation, Measurement Science and Technology, Volume 15, Issue 5, pp. 896-904, May 2004. DOI: $10.1088 / 0957-0233 / 15 / 5 / 017$

[20] Z. Liu, C. Cai, M. Yu, M. Dong, Testing of accelerometer transverse sensitivity using elliptical orbits, MAPAN 33 (2018), pp. 217-226.

[21] J. J. Dosch, D. M. Lally, Automated testing of accelerometer transverse sensitivity, 2000. Online [Accessed 11 June 2021] http://www.modalshop.com/techlibrary/JDosch $\% 20$ transverse $\%$ 20calibration.pdf 